\title{
Correction to: Sam68 Promotes Invasion, Migration, and Proliferation of Fibroblast-like Synoviocytes by Enhancing the NF-kB/P65 Pathway in Rheumatoid Arthritis
}

\author{
Weiwei Sun, ${ }^{1}$ Rongqing Qin, ${ }^{1}$ Rui Wang, ${ }^{2}$ Dazhi Ding, ${ }^{1}$ Zhaohui Yu, ${ }^{1}$ Yuxi Liu, ${ }^{1}$ Ruilong Hong, \\ Zhen Cheng, ${ }^{1}$ and Youhua Wang ${ }^{1,3}$
}

Correction to: Inflammation (2018) 41(5):1661-1670

https://doi.org/10.1007/s10753-018-0809-4

Unfortunately the author name, Rongqing Qin, was wrongly spelt as Rongqin Qin in the original version. It has been updated and the complete corrected author group is given below.

Weiwei Sun, Rongqing Qin, Rui Wang, Dazhi Ding, Zhaohui Yu, Yuxi Liu, Ruilong Hong, Zhen Cheng, and Youhua Wang.

Weiwei Sun and Rongqing Qin contributed equally to the original article. The online version of the original article can be found at https://doi.org/ 10.1007/s10753-018-0809-4

\footnotetext{
${ }^{1}$ Department of Orthopedics, Affiliated Hospital of Nantong University, No.20 Xisi Road, Nantong City, Jiangsu, 226001, China

${ }^{2}$ North Sichuan Medical College, Nanchong, 637000, China

${ }^{3}$ To whom correspondence should be addressed at Department of Orthopedics, Affiliated Hospital of Nantong University, No.20 Xisi Road, Nantong City, Jiangsu, 226001, China. E-mail: wangyouhua99@163.com
} 